\title{
Percutaneous Biportal Endoscopic Surgery for Lumbar Degenerative Diseases
}

\author{
Jung Hyun Lee, Kyung-Chul Choi, Hyeong Ki Shim, Seung Ho Shin, Dong Chan Lee \\ Department of Neurosurgery, The Leon Wiltse Memorial Hospital, Anyang, Korea
}

Corresponding Author: Dong Chan Lee, MD

Department of

Neurosurgery, The Leon

Wiltse Memorial Hospital, 560, Gyeongsu-daero,

Dongan-gu, Anyang 14112, Korea

Tel: $+82-31-460-1225$

Fax: +82-31-460-1224

E-mail: surgicel@hanmail.net

Received: January 12, 2017 Revised: June 15, 2017 Accepted: June 19, 2017
Objective: Percutaneous uniportal endoscopic surgery is comparable to conventional open surgery and maintains the surrounding structures, but it has restrictive indications and technical difficulties in sufficient decompression. Percutaneous biportal endoscopic surgery (PBES) can compensate the shortcomings and provide sufficient decompression safely. The purpose of this study is to introduce PBES technique and to report clinical outcome. Methods: Seventeen consecutive patients who underwent PBES for spinal stenosis or disc herniation were enrolled for this study. The technique was based on two routes; endoscopic view portal and working portal. Clinical outcomes were assessed using visual analogue scale (VAS) and modified Macnab's criteria. Results: There were 7 female and 10 male patients whose age ranged from 33 to 80 years old (mean 53 years). A total of 10 patients underwent decompression and discectomy for herniated intervertebral disc (HIVD), and 7 patients underwent laminectomy and foraminotomy for lumbar spinal stenosis. The mean follow up period was 8.3 months (6-12 months). The mean VAS scores for back pain preoperatively and postoperatively at the 1 month and 6 month followups were $6.6 \pm 1.8,2.8 \pm 2.0$, and $2.5 \pm 2.0$, respectively. The mean VAS scores for leg pain preoperatively

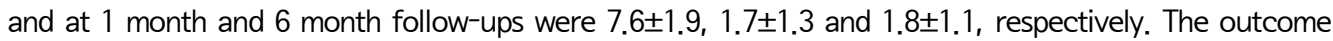
of the procedure was excellent in 8 patients and good in 9 patients according to the modified Macnab's criteria. Conclusion: PBES can achieve effective decompression and show comparable results with conventional open surgery.

Key Words: Percutaneous biportal endoscopic surgery, Minimal invasive spinal surgery, Spinal stenosis, Lumbar disc herniation

\section{INTRODUCTION}

Conventional surgical procedures for lumbar degenerative diseases, such as herniated intervertebral disc (HIVD) and spinal stenosis are microdiscectomy and laminectomy with foraminotomy respectively ${ }^{5,10,17)}$. Over time, minimal invasive surgery (MIS) has become the preferred treatment of lumbar degenerative diseases. Percutaneous endoscopic lumbar discectomy (PELD) is used as an alternative procedure to microdiscectomy for HIVD and bilateral decompression via a unilateral approach, and microendoscopic decompression has replaced conventional laminectomy and foraminotomy for lumbar spinal stenosis ${ }^{3,10,13)}$. Endoscopic surgery is comparable to conventional open surgery and maintains the surrounding structures, but it has restrictive indications and technical difficulties in sufficient decompre$\operatorname{ssion}^{16)}$. Although percutaneous endoscopic surgery via a uniportal route has been attempted for spinal stenosis and foraminal stenosis, it has inherent limitation, which can only remove precise target points in the narrow field. Percutaneous biportal endo- scopic surgery (PBES) can compensate the shortcomings and provide sufficient decompression safely. The authors will describe the PBES technique and introduce our experiences of treating patients who have lumbar degenerative diseases by PBES.

\section{MATERIALS AND METHODS}

\section{Patients}

A total of 17 patients with various lumbar degenerative diseases were treated using PBES between July 2015 and December 2015. The patient inclusion criteria were the diagnosis of lumbar canal stenosis without instability, HIVD and no previous surgery at the current site. All patients were admitted to the hospital for surgery. All procedures were conducted by 2 neurosurgeons who have many years of experience in conventional open surgery and percutaneous uniportal endoscopic surgery. Clinical outcomes were assessed using visual analogue scale (VAS) and modi- 
fied Macnab's criteria (Excellent: no pain, no restriction of mobility, return to normal work and level of activity, Good: occasional nonradicular pain, relief of presenting symptoms, able to return to modified work, Fair: some improved functional capacity, still handicapped and/or unemployed, Poor: continued objective symptoms of root involvement, additional operative intervention needed at the index level irrespective of length of postoperative follow-up).

\section{Surgical Technique}

Surgery was performed under epidural or general anesthesia. The patient was in the prone position with radiolucent Wilson frame for abdomen free and all bony prominences were gently

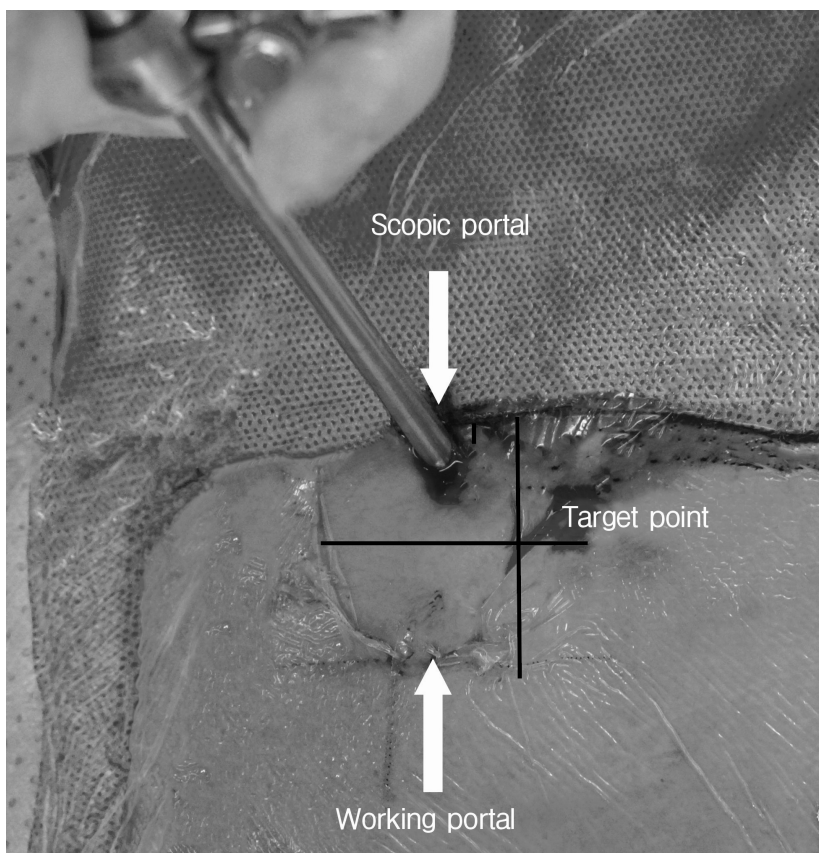

Fig. 1. The skin entry points. Cranial and caudal entry points were located at $1 \mathrm{~cm}$ above and below the target lesion. Also, each entry points were located $1 \mathrm{~cm}$ from the lateral of the target lesion. padded. A biplane C-arm fluoroscopy was used during the operation. The skin entry points differs from the target lesion(e.g. lower margin of the upper lamina, and lateral pars etc.) depending on the pathology. Cranial and caudal entry points were located $1 \mathrm{~cm}$ above and below the taget lesion. Alongside this, each entry points were located at $1 \mathrm{~cm}$ laterally of the target lesion. Most cranial entry point was used as the scopic portal and most caudal entry point was used as the working portal although, not always (Fig. 1). After fascia opening and blunt muscle splitting dissection, serial dilators were inserted (Fig. 2). After removal of dilators, an arthroscope (Conmed Linvatec, NY, USA, Fig. 2) was inserted into the scopic portal. At first, it is indispensable to find the osseus margin (e.g. spinolamina junction, lower margin of the lamina, or lateral pars, etc.). After hemostasis, soft tissues overlain the bone and interlaminar space are removed. A high speed burr (Primado2, Nakanishi Inc, Japan) may be used to thin the lamina. Following this, the lateral recess and foramen are decompressed. Subsequently, partial medial facetectomy is performed using a drill or Kerisson rongeur. Ligamentum flavum is carefully dissected from the dura and then removed with a Kerrison rongeur or pituitary forcep. It is possible to operate bilaterally though the unilateral access in the sense of an undercutting contralateral lamina technique using a drill and a Kerrison rongeur. Contralateral ligamentum flavum is also carefully dissected from the dura and then removed with a Kerrison rongeur or pituitary forcep. The decompression was considered complete when the thecal sac and roots (ipsilateral or contralateral) are clearly visible (Fig. 3). If discectomy is required, using a blunt nerve hook, the medial wall of the pedicle and the lateral aspect of the nerve root should be identified prior to discectomy. Following this, discectomy can be done after retraction of the nerve root. In case of paramedian approach, the transverse process, lateral aspect of isthumus and the lateral facet are confirmed. Drilling is taken up at the lateral pars and the superior articular process, and continues in a medial direction, to expose the foraminal ligament and the inferior aspect of the pedicle underlying the transverse process. The foraminal ligament is resected using a Kerrison rongeur. Further dissections expose the exiting root and the nerve is laterally displaced with a dissector. The herniated disc materials are then removed with a pituitary forcep. The high-frequency bipolar-current (Ellman Trigger-Flex probe, Ellman International Inc.,
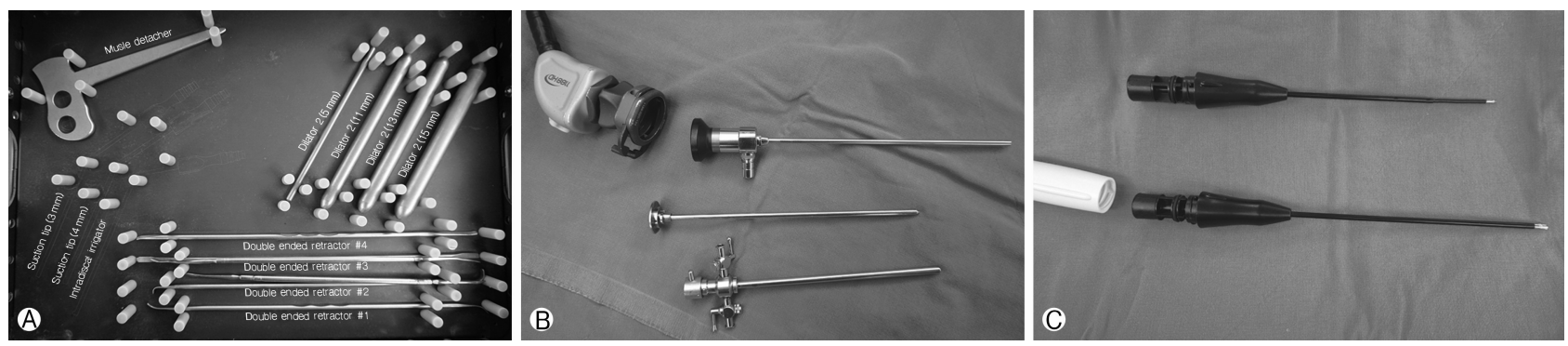

Fig. 2. (A) Dissector for exposure of the osseous margin and dilators for PBES. (B) Arthroscope (Conmed Linvatec, NY, USA). (C) Radiofrequency electrode (VAPR, DePuy Mitec, MA, USA) - useful equipment to halt bleeding in PBES. 
NY, USA) and radiofrequency electrode (VAPR, DePuy Mitec, MA, USA, Fig. 2) can be applied at the bleeding points. The bone wax also used for the bone bleeding. After indwelling drainage catheter was inserted at the operation site, the incision sites were closed.

\section{RESULTS}

Our subjects included 7 female and 10 male patients whose ages ranged from 33 to 80 years (mean 53 years). The mean operation time was 74 minutes (62-93 minutes) and the mean hospital days after surgery was 5.1 days (3-8 days). A total of 10 patients underwent decompression and discectomy for HIVD, and 7 patients underwent laminectomy and foraminotomy for lumbar spinal stenosis. The mean follow up period was $8.3 \pm 1.5$ months (6-12 months). The mean VAS scores for back pain pre-
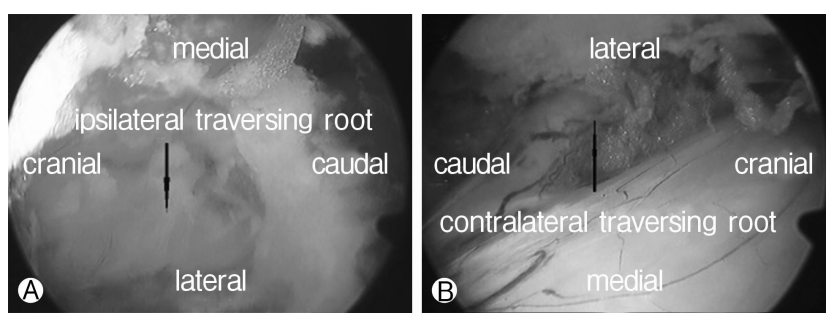

Fig. 3. Intraoperative endoscopic view. Enough decompression of the thecal sac, ipsilateral (A) and contralateral (B) nerve root was observed. operatively and postoperatively at the 1 month and 6 month follow-ups were $6.6 \pm 1.8,2.8 \pm 2.0$, and $2.5 \pm 2.0$, respectively. The mean VAS scores for leg pain preoperatively and at 1 month and 6 month follow-ups were $7.6 \pm 1.9,1.7 \pm 1.3$ and $1.8 \pm 1.1$, respectively. The outcome of the procedure was excellent for 8 patients and good in 9 patients according to the modified Macnab's criteria. The demographic data and clinical findings are summarized in Table 1. There were 2 reported procedure-related complications, such as cerebrospinal fluid leakage and postoperative hematoma. However, it did not affect the symptoms. All patients were discharged when the safety and symptoms had been relieved.

\section{Case}

A 66 year old female patient presented with lower back pain and both posterior leg pain. Her symptoms had been present for about 2 years and had been gradually deteriorated. Her symptoms were not relieved after the epidural block. MR images showed canal stenosis at the L4-L5 level (Fig. 4). The patient underwent unilateral laminectomy and bilateral foraminotomy via a left approach by PBES. Following surgery, her symptoms were relieved. Postoperative MR images showed decompression of stenotic canal (Fig. 4).

\section{Case}

A 46 year old female patient presented with both left buttock pain and left posterior leg radiating pain. Her symptoms had

Table 1. The demographic data of the 14 patients and clinical findings

\begin{tabular}{|c|c|c|c|c|c|c|c|c|c|}
\hline No. & Sex & Age & Level & Location & Operation & Op time & HD after Op & Complications & Macnab's criteria \\
\hline 1 & $M$ & 47 & L5-S1 & Right & PLD & 68 & 7 & & Good \\
\hline 2 & $\mathrm{~F}$ & 52 & L4-L5 & Left & PLD & 70 & 4 & & Excellent \\
\hline 3 & $M$ & 51 & L5-S1 & Left & PLD & 63 & 4 & & Excellent \\
\hline 4 & $M$ & 33 & L4-L5 & Right & PLD & 69 & 5 & & Good \\
\hline 5 & $M$ & 50 & L4-L5 & Right & PLD & 62 & 3 & & Excellent \\
\hline 6 & $\mathrm{~F}$ & 66 & L4-L5 & Left & PLF (UBF) & 76 & 5 & & Good \\
\hline 7 & $\mathrm{~F}$ & 39 & L4-L5 & Left & PLF (UBF) & 71 & 6 & & Excellent \\
\hline 8 & $M$ & 58 & L4-L5 & Left & PLF (UBF) & 82 & 4 & & Good \\
\hline 9 & $\mathrm{~F}$ & 46 & L5-S1 & Left & $F \& D$ & 93 & 7 & Dura tear & Excellent \\
\hline 10 & M & 57 & L4-L5 & Left & PLD & 75 & 5 & Hematoma & Good \\
\hline 11 & $\mathrm{~F}$ & 52 & L4-L5 & Right & PLF & 62 & 3 & & Excellent \\
\hline 12 & M & 59 & L4-L5 & Left & $F \& D$ & 70 & 4 & & Excellent \\
\hline 13 & M & 47 & L3-L4 & Right & PLD & 76 & 5 & & Good \\
\hline 14 & $\mathrm{~F}$ & 80 & L4-L5 & Right & PLF (UBF) & 87 & 7 & & Good \\
\hline 15 & F & 78 & L3-L4 & Left & PLF (UBF) & 84 & 7 & & Excellent \\
\hline 16 & M & 33 & L4-L5 & Right & PLD & 70 & 4 & & Good \\
\hline 17 & $M$ & 58 & L3-L4 & Left & PLF (UBF) & 92 & 8 & & Good \\
\hline
\end{tabular}

PLD: partial lamectomy and discectomy, PLF: partial laminoforaminotmy, UBF: unilateral approach and bilateral foraminotomy, F\&D: foraminotomy \& discectomy, Op: operation, HD: hospital day 
persisted for the prior months and it had aggravated about 1 month ago. Preoperative MR images showed herniation of nucleus pulposus at the L5-S1 level (Fig. 5). The patient underwent partial laminectomy and discectomy via a left approach by PBES. Following surgery, her symptoms were relieved. Postoperative MR images showed the removal of herniated disc materials (Fig. 5).

\section{Case}

A 47 year old male patient presented with both lower back pain and right buttock pain. Manual muscle test of ankle dorsiflexion was grade IV. His symptoms were triggered 7 days prior to admission, occurring suddenly during his work. Initial MR images showed disc rupture with downward migration at the L3-L4 level (Fig. 6). The patient underwent partial laminectomy and discectomy via a right approach using PBES. After surgery his symptom were relieved. Postoperative MR images showed the removal of herniated disc materials (Fig. 6).

\section{DISCUSSION}

Many MISs constantly innovate and new MIS have been created. PELD was first introduced in the early 1980s, and was then widely performed in the late 1990s including transforaminal and interlaminar approach for the treatment of HIVD ${ }^{1,12)}$. As the surgical method gradually evolved, surgical indications have become wider and its outcomes have become comparable to conventional open surgery ${ }^{13)}$.

However, there are still restrictions that include narrow neural foramen, high iliac crest, high canal compromise, high-grade migration, presence of large vessels in the neural foramen, and narrow interlaminar space ${ }^{4,6-8,13,14)}$. There remain to be limited applications in definite stenosis although some reports asserted that it can be used in the operation of spinal canal senosis ${ }^{9,15}$. Working space and various pieces of epuipment are restricted in the uniportal system.

Bilateral decompression with a unilateral approach under the microscope as MIS are good procedure for lumbar spinal stenosis. By preserving the normal structures, a bilateral decompression via a unilateral approach may decrease the incidence of delayed instability ${ }^{11)}$. This technique is commensurate with the results of conventional techniques while minimizing traumatization. However, this technique cannot avoid the injury of ipsilateral paraspinal muscle and interspinous ligament.

PBES could compensate for these shortcomings with its associated advantages. First of all, since the lens of scope can locate in front of the target point and there is no equipment inside the scope, direct visualization is possible to the contralateral side preserving the interspinous ligament with minimized muscle injury. Secondly, conventional equipments can be freely used, and so sufficient bone work is possible as much as conventional open surgery. Thirdly, as it is possible to retract neural tissue using one portal route, manipulation is more easily and safely carried out by equipments through the working portal than
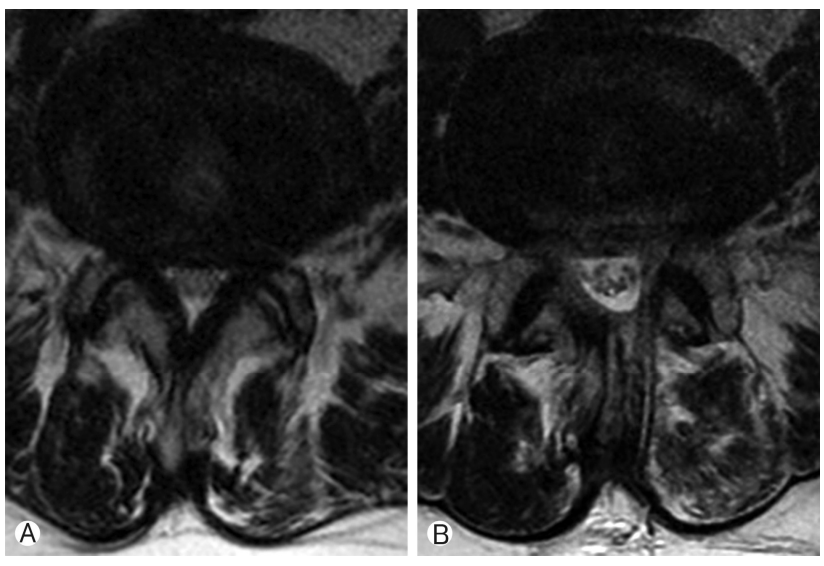

Fig. 4. (A) Preoperative MR image showed canal stenosis at the L4-L5 level. (B) Postoperative MR image showed decompression of spinal canal.
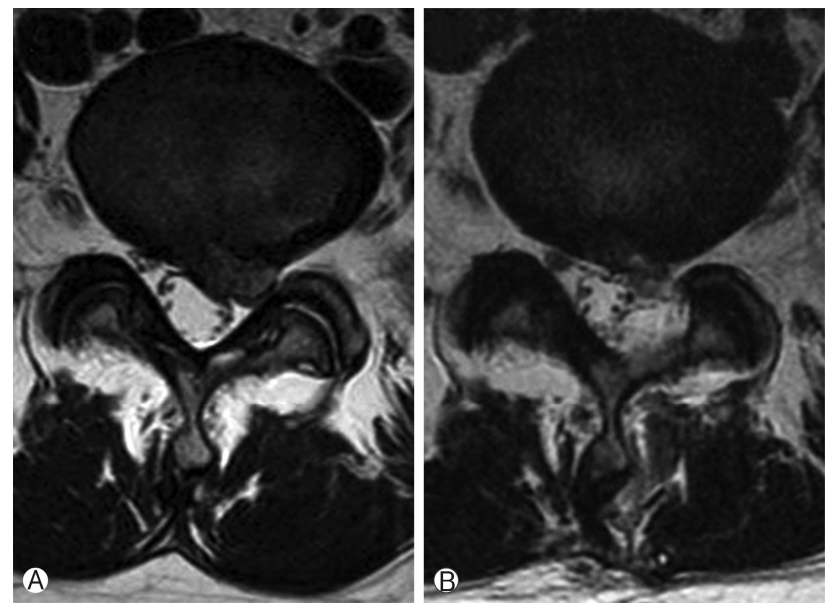

Fig. 5. (A) Preoperative MR image showed herniation of nucleus pulposus at the L5-S1 level. (B) Postoperative MR image showed the removal of herniated disc materials.
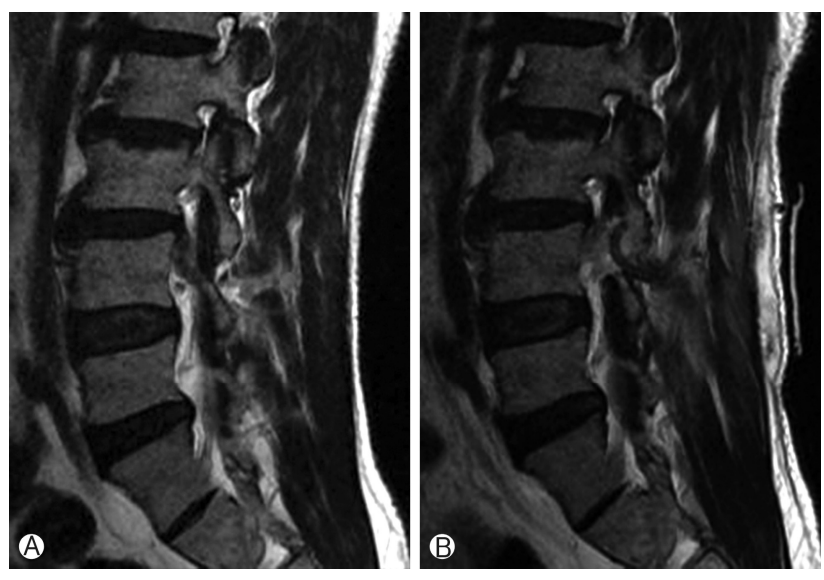

Fig. 6. A Preoperative MR image showed disc rupture with downward migration at the L3-L4 level. (B) Postoperative MR image showed removal of herniated disc materials. 
the uniportal endoscopic surgery.

We experienced some complications related to the operation as follows unrecognized dural tear and postoperative hematoma accumulation. Among these complications, dura tear is caused by low experience, which with copious experience these can be overcome. As PBES takes more bone resection compared to uniportal endoscopic surgery, the bone bleeds more, which causes postoperative hematoma. Despite the fact that the bipolar current, radiofrequency electrode, and bone wax were used to that hemostasis, hemostasis is so difficult to manage in some cases. Some studies advocate that drainage is not necessary, although our study recommends drainage.

There are some considerations to be made during the procedure. First of all, in the operation field saline needs to flow continuously to prevent increasing the epidural hydrostatic pressure and intradiscal pressure, whilst also preventing thermal injury. It also provides a clear vision and hemostatic effect. For the proper circulation of saline, it is important to use techniques which indwell a tube and maintains pump pressure to $25-30 \mathrm{mmHg}^{2}$. Secondly, because of the freely movable scope and the location of the lens, this procedure allows excellent visualization of the ipsilateral and contralateral sides. Thereby the surgeon can often overestimate the degree of decompression. Since the thecal sac and the nerve root seem to be highly decompressed with a little bit of bone resection, it may not be sufficient decompression. It can be solved by more experiences. Finally, hemostasis is difficult to manage, especially when bone bleeding occurs. We recommend using a drainage catheter.

The technique is similar to open microsurgery and the learning curve is not too steep. It can facilitate neural retraction for disc removal while one portal endoscopic surgery has a limitation of neural retraction. In spite of the short observation period, the current paper demonstrates the good results of PBES for lumbar degenerative diseases. We consider this procedure can be a sufficient and safe alternative to open surgeries for lumbar degenerative diseases.

\section{CONCLUSION}

PBES can achieve effective decompression and show comparable results with conventional open surgery as a minimal invasive spinal technique.

\section{REFERENCES}

1. Brayda-Bruno M, Cinnella P: Posterior endoscopic discectomy (and other procedures). Eur Spine J Suppl 1:S24-29, 2000

2. Choi CM, Chung JT, Lee SJ, Choi DJ: How I do it? Biportal endoscopic spinal surgery (BESS) for treatment of lumbar spinal stenosis. Acta Neurochir (Wien) 158:459-463, 2016

3. Choi KC, Kim JS, Park CK: Percutaneous endoscopic lumbar discectomy as an alternative to open lumbar microdiscectomy for large lumbar disc herniation. Pain Physician 19:291-300, 2016
4. Choi KC, Lee JH, Kim JS, Sabal LA, Lee S, Kim H, et al: Unsuccessful percutaneous endoscopic lumbar discectomy: a singlecenter experience of 10,228 cases. Neurosurgery 76:372-380, 2015

5. Costa F, Sassi M, Cardia A, Ortolina A, De Santis A, Luccarell G, et al: Degenerative lumbar spinal stenosis: analysis of results in a series of 374 patients treated with unilateral laminotomy for bilateral microdecompression. J Neurosurg Spine 7:579-586, 2007

6. Kim CH, Chung CK, Woo JW: Surgical outcome of percutaneous endoscopic interlaminar lumbar discectomy for highly migrated disc herniation. Clin Spine Surg 29:259-266, 2007

7. Lee S, Kim SK, Lee SH, Kim WJ, Choi WC, Choi G, et al: Percutaneous endoscopic lumbar discectomy for migrated disc herniation: classification of disc migration and surgical approaches. Eur Spine J 16:431-437, 2007

8. Lee SH, Kang BU, Ahn Y, Choi G, Choi YG, Ahn KU, et al: Operative failure of percutaneous endoscopic lumbar discectomy: a radiologic analysis of 55 cases. Spine (Phila Pa 1976) 31:285-290, 2006

9. Komp M, Hahn P, Merk H, Godolias G, Ruetten S: Bilateral operation of lumbar degenerative central spinal stenosis in fullendoscopic interlaminar technique with unilateral approach. J Spinal Disord Tech 24:281-287, 2011

10. Mobbs RJ, Li J, Sivabalan P, Raley D, Rao PJ: Outcomes after decompressive laminectomy for lumbar spinal stenosis: comparison between minimally invasive unilateral laiminectomy for bilateral decompression and open laminectomy: clinical article. J Neurosurg Spine 21:179-186, 2014

11. Poletti CE: Central lumbar stenosis caused by ligamentum flavum: unilateral laminotomy for bilateral ligamenetectomy: Preliminary report of two case. Neurosurgery 37:343-347, 1995

12. Ruetten S, Komp M, Godolias G: An extreme lateral access for the surgery of lumbar disc herniations inside the spinal canal using the full-endoscopic uniportal transforaminal approach. Technique and prospective results of 463 patients. Spine (Phila Pa 1976) 30:2570-2578, 2005

13. Ruetten S, Komp M, Merk H, Godolias G: Full-endoscopic interlaminar and transforaminal lumbar discectomy versus conventional microsurgical technique: a prospective, randomized, controlled study. Spine (Phila Pa 1976) 33:931-939, 2008

14. Ruetten S, Komp M, Merk H, Godolias G: Recurrent lumbar disc herniation after conventional discectomy: a prospective, randomized study comparing full-endoscopic interlaminar and transforaminal versus microsurgical revision. J Spinal Disord Tech 22:122-129, 2009

15. Ruetten S, Komp M, Merk H, Godolias G: Surgical treatment for lumbar lateral recess stenosis with the full-endoscopic interlaminar and transforaminal approach versus conventional microsurgical technique: a prospective, randomized, controlled study. J Neurosurg Spine 10:476-485, 2008

16. Shin KH, Chang HG, Rhee NK, Lim KS: Revisional percutaneous full endoscopic disc surgery for recurrent herniation of previous open lumbar discectomy. Asian Spine J 5:1-9, 2011

17. Yorimitsu E, Chiba K, Toyama Y, Hirabayashi K: Long-term outcomes of standard discectomy for lumbar disc herniation: a follow-up study of more than 10 years. Spine (Phila Pa 1976) 26:652-657, 2001 